ainsi que la tension entre les électrodes : les meilleurs résultats furent obtenus pour o, 3 ampères et 1 roo volts. Sous ces conditions, et en admettant les bases du calcul de Crookes, si l'on employait de l'air enrichi en oxygène par la liquéfaction, il serait possible d'établir la tonne de nitrate à moins de I Io francs, prix extrêmement séduisant.

Ce ne sont là, malheureusement encore, que des chiffres théoriques. Crookes semble trop compter sans les difficultés d'ordre pratique que présente la liquéfaction industrielle de l'air. D'autre part, malgré les promesses de leurs premières expériences, Mac Douglas et Howles ne sont pas encore en état d'entamer des essais industriels, car ils ne réussirent à actionner une série de leurs appareils par un seul alternateur, qu'au prix d'une perte considérable d'énergie, dans une foule de régulateurs, et sans obtenir une marche régulière.

Ainsi, l'industrie électrochimique des nitrates n'est pas encore créée : mais les résultats auxquels ont conduit les études déjà faites montrent assez qu'elles doivent d'être poursuivies et élargies. Ces résultats donnent à prévoir que les applications pratiques ne dépendent plus que de l'invention de dispositifs économiques et sûrs, devant permettre un meilleur emploi de l'énergie.

Et, pour peu que l'activité des électrochimistes réponde à l'importance du problème qui leur est posé, il n'y a aucune exagération à lui prédire une solution prochaine; celleci est moins complexe d'ailleurs, que celle de la production $\mathrm{du}$ fer, où les électrométallurgistes touchent presque au but; et alors que les moindres essais d'électrométallurgie entrâ̂nent des dépenses qui se comptent par centaines de mille francs, il apparaît que les dépenses relatives aux essais de fabrication des nitrates ne doivent se chiffrer que par quelques milliers de francs.

Il est à désirer que pour hâter l'avènement d'une telle industrie qui, à nulle autre pareille, intéresse l'avenir industriel de notre pays, les propriétaires des forces naturelles tentent quelques efforts. Les travaux de laboratoire dont nous venons de parler valent la peine d'être reproduits sur une plus grande échelle et nous sommes convaincus qu'ils l'auraient été déjà si, pour des expériences de ce genre, il était facile de trouver de l'énergie électrique à bon compte.

\section{P. Pierron,}

Chef des travaux

à l'Ecole de Chimie industrielle de Lyon

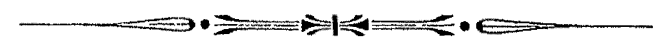

\section{Les desiderata à réaliser dans la réduction des minerais de fer au four électrique.}

La Métallurgie ne pouvait manquer de participer au mouvement en avant de l'industrie électrotechnique. On a commencé et, du reste, mené à bien, la fabrication courante des fontes de fer réactives, alliées dans des proportions déterminées à toute une série de métaux rares, devant donner des propriétés nouvelles aux aciers industriels fabrim qués en grandes masses dans les usines importantes. On peut compter, dans ces métaux alliés, comme dans ceux qui sont déjà dans les transactions commerciales en France,et surtout en Allemagne et en Angleterre, les fontes au chrôme, au nickel, au molybdène, au tantale, au titane, au tungstène, au vanadium. Ceux sur lesquels des essais sont encore à l'étude sont les fontes au cobalt, à l'iridium, à l'osmium, au palladium et au rhodium; enfin l'aluminium est obtenu en grand presque à l'état de pureté. Mais, ainsi que le fait remarquer, ici même, M. Pierron, l'électrochimie ne pouvait se restreindreà la fabrication des réactifs métallurgiques, vu la petite quantité de matière employéc et, par suite, vu l'exiguité de son marché industriel. II fallait donc viser plus haut et, actuellement, il ne s'agit rien moins que de prendre les lieu et place du haut-fourneau.

Nous arrivons donc à nous demander quelles sont les conditions désirables à réaliser pour atteindre ce but; mais avant dè les formuler, il faut d'abord connaître les résultats obtenus par les moyens dont on dispose actuellement.

\section{I}

On sait que les corps qui s'unissent le plus facilement au fer et qui forment avec lui les combinaisons les plus stables, sont l'acide carbonique, l'oxygène, le soufre et le phosphore. Mais ce sont surtout les deux premiers qui constituent avec le fer la grande majorité des matières dont on cherche à isoler le métal. On tâche donc de chasser de ces corps complexes, soit leur acide carbonique, soit leur oxygène et, pour cela, on cherche un autre corps dont l'affinité pour ces derniers éléments soit plus grande que pour le fer qui doit être séparé. Cette opération est la réduction et, jusqu'à présent, le corps réducteur le plus employé, en présence de la chaleur, est le carbone.

Le haut-fourneau est l'appareil destiné à cet objet. Donc, les matières qui entrent dans l'appareil sont : les corps à réduire, le corps réducteur et l'agent oxydant devant donner, avec une partie du corps réducteur, la quantité de chaleur nécessaire à l'opération. D'un autre côté, les matières qui sortent du haut-fourneau sont : le métal réduit,combiné dans une certaine proportion, variable du reste, au corps réducteur; les matières fondues séparées du métal et les gaz résultant de l'opération.

Les matières les plus généralement employées sont les minerais de fer proprement dits sous deux états: $1^{0}$ les minerais carbonatés ; $2^{0}$ les oxydes très divers.

Les minerais carbonatés sont,en général : le fer carbonaté spathique; les sphérosidérites; l'hématite brune, sesquioxyde ou carbonate de protoxyde de fer; l'hématite brune mamelonnée; l'hématite brune ordinaire; la minette oolithique souvent très phosphoreuse.

Les oxydes les plus employés sont :

L'hématite rouge $\mathrm{Fe}^{2} \mathrm{O}^{3}$, le minerai magnétique de protoxyde et de peroxyde de fer $\mathrm{Fe}^{3} \mathrm{O}^{4}$. Restent les sulfures de fer ou pyrites, mais qu'il est nécessaire de désulfurer pour les faire passer à l'état d'oxydes avant leur emploi ; ce n'est done pas une matière traitée directement. Fn plus, tous ces 
carbonates et même ces oxydes de fer subissent, suivant les cas, l'opération du grillage; ils entrent dans le lit de fusion soit à l'état cru, soit grillés. Nous ne parlerons donc pas de ces opérations préliminaires qui se trouvent dans tous les traités de métallurgie; ce que nous retiendrons surtout, c'est que le haut-fourneau doit séparer le fer des corps qui lui sont unis.

Il ne faut pas perdre de vue que, le plus souvent, les minerais seuls ne suffisent pas pour que le phénomène de la réduction se fasse convenablement. On est obligé de leur ajouter une classe de matières diverses, connues sous le nom de fondants et, suivant la composition chimique du minerai, ce fondant est choisi de telle sorte qu'il facilite la fusibilité des matières résultant de la fusion réductive. Naturellement, la composition et la proportion du rondant variera suivant le ou les minerais employés et le produit que l'on se propose d'obtenir; ces matières n'interviennent que rarement dans le phénomène de la fusion en tant que corps combustibles.

Enfin, l'agent réducteur le plus employé est le carbone, à l'état de charbon de bois, de houille crue, quoique beaucoup plus rarement, de coke presque toujours au moins en Europe, et d'anthracite surtout en Amérique. Pour mémoire, on a essayé la réduction des minerais de fer au moyen des hydrocarbures liquides et gazeux, mais nous ne connaissons pas les résultats de ces expériences.

En plus, l'élément oxydant indispensable à la production calorifique est l'air injecté dans le fourneau, à une pression variant de $0^{k}, 030$ à $1^{k}$ par centimètre carré.

Les matières produites par l'appareil sont : le fer combiné au carbone, ou fonte; les laitiers, résultat de la fusion des matières séparées du fer, et les gaz, acide carbonique, oxyde de carbone, azote, vapeur d'eau pouvant être considérée comme un gaz à cette température, venant de l'air et du minerai s'il en contient, ce qui est généralement le cas. Donc, une partie du carbone forme les combinaisons solides et gazeuses de la réduction, le surplus donne la quantité de chaleur nécessaire à cette opération.

Une circonstance très favorable aux bons résultats des opérations de réduction réside dans le sens de la marche des diverses matières sur lesquelles se fait la combinaison. En effet, les matières solides devant se combiner sont déversées au sommet de l'appareil, elles marchent donc de haut en bas; d'un autre côté les éléments devant provoquer la réduction, avant la fusion, sont les gaz chauds provenant de la combustion du carbone qui cheminent de bas en haut en sens inverse des matières à réduire; le carbone descendant est chauffé par eux ainsi que les minerais, et cet ensemble subit une préparation avantageuse pour le résultat final. On peut dire que la fusion réductive est méthodique, ainsi que la transmission calorifique; c'est certainement là le fait capital auquel est due la bonne utilisation de la chaleur nombre de fois constatée dans la marche des fourneaux.

Nous ferons encore remarquer que les matériaux qui entrent dans la construction du fourneau agissent aussi pendant l'opération de la fusion réductive comme agents chimiques. Ainsi, par exemple, s'il s'agissait de traiter une matiere dans laquelle les bases, comme la chaux, la

\section{HAUT FOURNEAU TYPE DU LUXEMBOURG}
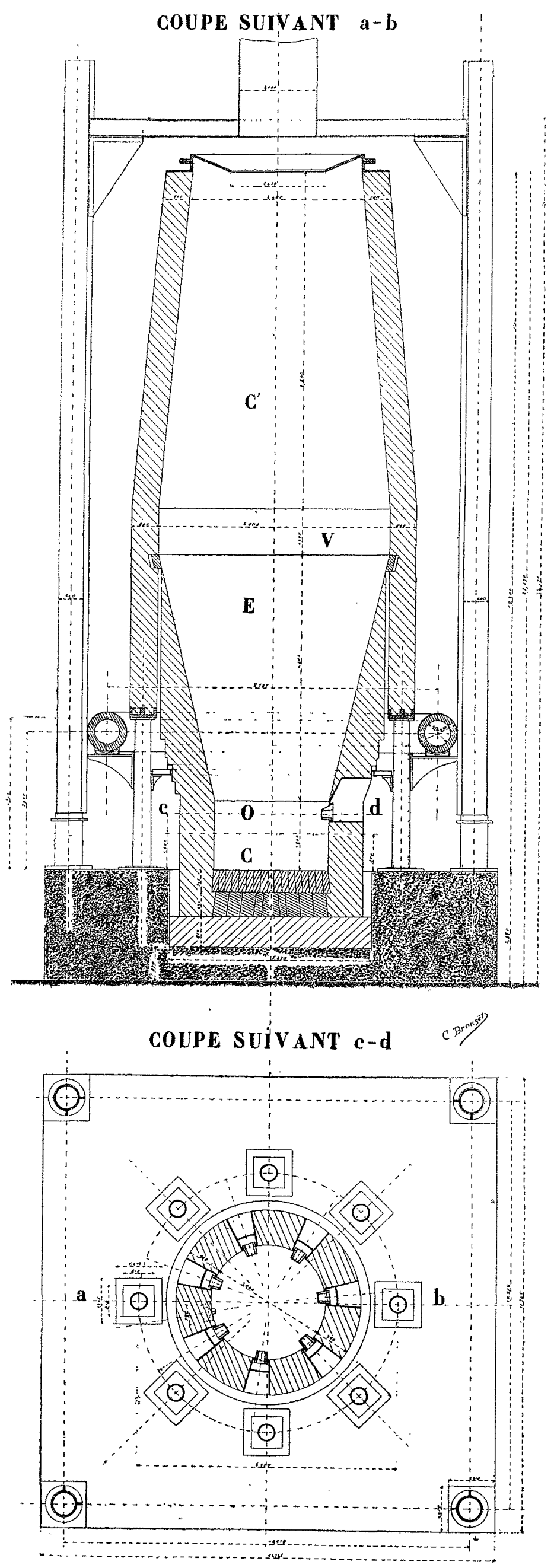
magnésie, voire même l'alumine dans certains cas, seraient en grandes proportions, il serait nécessaire que les matériaux du four aient une composition analogue, autrement dit fussent basiques; si, au contraire, les matières précitées contenaient une proportion prépondérante de silice laquelle, dans le cas particulier, remplirait une fonction acide, les matériaux de construction du four devraient être acides. Si l'on n'observait pas ces conditions, on risquerait de voir les matériaux du four et les matières à traiter former des combinaisons très fusibles et, sous l'influence de la haute température, l'appareil serait rapidernent hors de service.

\section{II}

Tout le monde sait comment est composé un hautfourneau. Toutefois, pour donner une idée des dimensions colossales qu'atteignent généralement ces appareils, nous donnons ci-contre la photogravure d'un avant-projet de haut-fourneau suivant le type actuellement en marche dans le grand duché de Luxembourg.

La partie $C^{\prime}$, la cuve, a $8^{m} 800$ de hauteur, $4^{m} 400$ de diamètre intérieur au gueulard et 3 metres de rayon au grand diamètre; le ventre, $V$, dans sa partie cylindrique a le même diamètre et une hauteur de ${ }^{2} m_{2} 00$; les étalages, $\mathrm{E}$, ont $6^{\mathrm{m}} 400$ de haut et $\mathrm{I}^{\mathrm{m}} 500$ de rayon à leur réunion avec l'ouvrage et le creuset O.C. qui constituent un cylindre de 1 m800 de hauteur. Ainsi les dimensions intérieures de cet appareil sont : hauteur $18 \mathrm{~m}^{2} 200$, plus grand diametre: (i) mètres. Extéricurement, ses dimensions sont de $7^{\text {m60o }}$ au plus grand diamètre et de $21^{\mathrm{m}} \mathrm{O} 7$ de hauteur comptée du sol au gueulard.

Le fourneau marche à gueulard fermé; on voit la prise de gaz au sommet et le cône de déchargement. Nous pensons que le système de fermeture adopté, variable avec chaque appareil est celui désigné en Angleterre sous le nom de Cup and Cone. $I 1$ y a 7 tuyères à l'ouvrage, et le creuset comporte un trou de coulée du laitier et un trou de coulée du métal, le tout à circulation d'eau pour la réfrigération. Observons aussi que, quoique traitant des matières basiques difficiles à maintenir liquides, le fourneau n'est pas à poitrine ouverte; donc, si l'on ne craint pas les accrochages, cela doit tenir, pour une part, à la température élevée du vent.

Le volume intérieur du fourneau étant de $34_{4}^{m 3} 404$ nous pouvons donner les renseignements suivants : la production de fonte par 24 heures est de $1 i 5.008^{k}$; le lit de fusion rendrait $35,5 \%$, d'où il résulterait que, par heure, on aurait $4.79^{2 \mathrm{k}}$ de fonte et par mètre carré de section de l'ouvrage et par heure $\frac{4.792}{7.0683}=677^{\mathrm{k}} 9$. La production par h eure du laitier très basique serait de $7.460^{k}$ et la consommation de coke de $4.855 k$. Il est bien évident que nous n'avons pas pu vérifier ces chiffres, nous ne les acceptons donc que sous toutes réserves.

Maintenant, si l'on ne considere que la construction du fourneau sans les appareils à air chaud et sans machines soufflantes, on arrive à voir que la maçonnerie réfractaire compte pour $1.335^{\mathrm{m}^{3}} \mathrm{r} 32$ et en poids 2.404 tonnes; la fonte y entrerait pour 80 tonnes et la tôle d'acier pour 150 . En résumé, la construction reviendrait en bloc i fr. 489.000 dans le pays Luxembourgeois.

Nous ne dirons rien des phénomènes de fusion réductive qui ont lieu dans cet appareil et dont on trouve la théorie dans tous les traités de métallurgie. Nous donnerons, toutefois, ce qui nous paraît indispensable, une balance de chaleur fournie par M. le professeur Ledebur de Freiberg pour un fourneau produisant de la fonte grise. Cela constituera une indication réelle au point de pue de la répartition catorifique et les électro-chinistes pourront peut-être avoir là des données pour de futurs appareils de réduction.

Le fourneau produisait $63.000^{k}$ de fonte grise en 24 heures; il a 23 mètres de hauteur et une capacité de $584^{\mathrm{m} 3}$.

\section{Élingevts de ha balance de chaleur}

Pour produire $\mathrm{I}^{\mathrm{k}}$ de fonte on consommait :

${ }_{1} k_{100}$ de coke contenant $92,5 \%$ de carbone soit, en carbone, $I^{k}$ or 7 .

$2^{\mathrm{k}} 440$ de minerai grillé ou le fer était à l'état de $\mathrm{Fe}^{2} \mathrm{O}^{3}$.

$0^{k} 625$ de castine contenant $43 \%$ d'acide carbonique ou $0^{k} 7^{3}$ de carbone et $0^{k} 197$ d'oxygène formant ensemble ok 270 de $\mathrm{CO}^{2}$.

On produisait $t^{k}+80$ de laitiers; le rapport de $\frac{\mathrm{CO}^{2}}{\mathrm{CO}}$ était de 0,542 .

La composition de la fonte était :

$\mathrm{C}, 3,4 ; \mathrm{Si}, \mathrm{I}, 2 ; \mathrm{Mn}, 0,5 ; \mathrm{Ph}, 1,3 ; \mathrm{Fe}, 93,6$. Total: 100.00.

La quantité de carbone introduite dans le haut-fourneau par kilog. de fonte était:

Carbone provenant du coke, $\mathrm{I}_{\mathrm{k}} 17$ )

Carbone provenant de $\left.\mathrm{CO}^{2} \mathrm{O}_{0} \mathrm{O}_{3}\right\}$ ikoyo

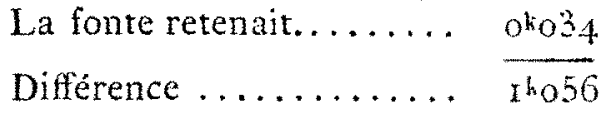

différence qui se retrouve dans les gaz du gueulard.

La quantité d'oxyde de carbone contenue dans ces gaz est donnée par la formule

$$
p=\frac{3}{7} y+\frac{3}{11} m y \quad \text { d'ou } \quad y=\frac{77 p}{33+21 m}
$$

Dans ces expressions :

$p$ représente le poids de carbone par kilog. de fonte produite;

$\because$ le poids d'oxyde de carbone;

$m$ le rapport $\frac{\mathrm{CO}^{2}}{\mathrm{CO}}$ déterminé par l'analyse ;

my le poids de l'acide carbonique.

On a done, dans le cas qui nous occupe:

$$
\begin{aligned}
& y=\frac{77 \times 1056}{33+2 \mathrm{I} \times 0^{\mathrm{k} .542}}=1^{\mathrm{k} .83 \mathrm{I}} \\
& \text { et } m y=0.542 \times 1.83 \mathrm{I}=\mathrm{o}^{\mathrm{k} .992} .
\end{aligned}
$$

On peut alors apprécier la quantité de gaz produite et celle d'air insuffté par kilog. de fonte. On a, en effet:
${ }_{1} \mathrm{k} 831$ de CO qui contient
$o^{k} 99^{2}$ de CO qui contient
Le gaz renferme donc.... $\overline{1,7667}$ d'oxygène 
Examinons maintenant la quantité d'oxygène que donne le lit de fusion.

L'acide carbonique de la castine a apporté $\mathrm{O}^{\mathrm{k}} \mathrm{I} 97$ d'oxygène r k337 de $\mathrm{Fe}^{2} \mathrm{O}^{8}$ produisant $\mathrm{Fe}=\mathrm{o}_{9} \mathrm{k} 36$ a apporté $\mathrm{o}^{\mathrm{k}} 4 \mathrm{O} 1$ d'oxygène $0^{k_{0} 25}$ de $\mathrm{Si} \mathrm{O}^{2}$ produisant $\mathrm{Si}=0^{\mathrm{k}_{\mathrm{O}} 12}$ a apporté oko 3 d'oxygène ${ }^{k_{0}} 007$ de $\mathrm{Mn}^{2} \mathrm{O}^{4}$ produisant $\mathrm{Mn}=0^{k} 005$ a apporté $\mathrm{O}^{\mathrm{k}} \mathrm{OO} 2$ d'oxygène $\mathrm{o}^{\mathrm{k}} \mathrm{O} 29$ de $\mathrm{Ph}^{2} \mathrm{O}^{5}$ produisant $\mathrm{Ph}=\mathrm{o}^{\mathrm{k}_{0} \mathrm{I}} 3$ a apporté $\mathrm{o}^{\mathrm{k}} \mathrm{o} 6 \mathrm{~d}$ d'oxygène Total de l'oxygène provenant du lit de fusion $\mathrm{O}^{\mathrm{k} 629}$ d'oxygène

L'air a donc apporté ${ }_{1}{ }^{k} 6_{7}-0^{k} 629={ }_{1} k_{1} 38$ d'oxygène correspondant à

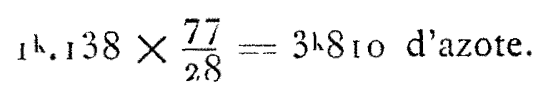

On a insufflé, pour obtenir $1^{h}$ de fonte :

$$
1^{\mathrm{k}} \mathrm{3} 38+3 \mathrm{k} 8 \mathrm{10}=4^{\mathrm{k}} 94^{8}
$$

et les $6^{\mathrm{k}} 633$ de gaz du gueulard étaient composés comme suit :

$\mathrm{CO}, \mathrm{l}^{\mathrm{h} 83 \mathrm{I}}$; $\mathrm{CO}^{2}, \mathrm{o}^{\mathrm{k}} 99_{2}$ et $\mathrm{Az}, 3^{\mathrm{k}} 810$; au total $6 \mathrm{k} 633$, en supposant, bien entendu, ce gaz exempt de vapeur d'eau.

On peut maintenant établir le compte des chaleurs reçues et dépensées.

$$
\text { io Chaleurs reçues }
$$

(a) Combustion du carbone :

Par kilog de fonte produite, les gaz du geulard contenaient okg92 d'acide carbonique, dont ok 270 provenaient du lit de fusion; l'excédent, soit ok7:2, étaient dus à la combustion de ok 197 de carbone.

Le carbone total brûlé était égal à $1^{\mathrm{k} o{ }_{7}} 7-\mathrm{ok}^{\mathrm{k}} 3_{4}=\mathrm{ok}_{9} 83$, dont olis 197 passaient à l'état de $\mathrm{CO}^{2}$, et le reste, oh 786 , était transformé en oxyde de carbone.

On doit calculer ainsi la chaleur développée :

Pour la production de $\mathrm{CO}^{2} \mathrm{ok}_{\mathrm{i}} \mathrm{97} \times 8.080=1.59 \mathrm{i}$ Pour la production de $\left.\mathrm{CO}{ }^{\mathrm{k}} 7 \mathrm{7} 86 \times 247^{3}=1.943\right\} 3.534$

(b) Chaleur provenant de l'air chaud:

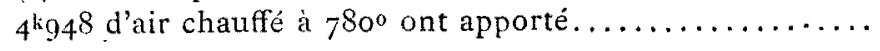

Total de la chaleur reçue ................ . $\overline{4.448}$

$$
2^{\circ} \text { Chaleurs dépensées }
$$

(a) Pour la réduction du minerai :

calories

okg36 de $\mathrm{Fe}$ provenant de $\mathrm{Fe}^{2} \mathrm{O}^{4}$ ont exigé ok $936 \times 1.796=\mathrm{r} .68 \mathrm{r}$ okol 2 de Si provenant de $\mathrm{SiO}^{2}$ ont exigé okol $2 \times 7.830=$ $\mathrm{o}^{\mathrm{k}} 005 \mathrm{de} \mathrm{Mn}$ provenant de $\mathrm{Mn}^{3} \mathrm{O}^{4}$ ont exigé $\mathrm{o}^{\mathrm{k} 005} \times 2.000=$ okor 3 de $\mathrm{Ph}$ provenant de $\mathrm{Ph}^{2} \mathrm{O}^{5}$ ont exigé okol $3 \times 5.700=$

Total des calories de la réduction............. 1.860

calories

(b) Chaleur emportée par la fonte................ 280

(c) Chaleur emportée parles laitiers..... $1 \mathrm{k} 480 \times 500=740$

(d) Chaleur emportée par les gaz $6 \mathrm{k} 663 \times 412 \times 0.237(1)=647$

(e) Chaleur absorbée par la vaporisation de l'eau' à $4 \mathrm{r}^{\circ}(2) \quad 102$

(f) Chaleur absorbée par la décomposition de la castine... $\quad 25_{4}$

$(g h)$ Refroidissements, tuyères et pertes $, \ldots \ldots \ldots \ldots \ldots \ldots, 465$

Total des chaleurs dépensées.............. $\overline{4.44}$

(1) Cette chaleur est considérée comme utilisée au chauffage du vent et non pas perdue.

(2) En admettant que le minerai contienne $4 \%$ d'eau hygrométrique et le coke $2,5 \%$, on avait à vaporiser : oko97 +oko28 $=0^{k}{ }_{2} 5$ grammes d'eau qui exigeaient $78 \mathrm{C} .+24 \mathrm{C} .=102$ calories.
Quel est maintenant le rendement calorifique de cet appareil?

Nous avons pour la chaleur affectivement utilisé : $4.448 \mathrm{C}$. reçues -465 C. perdues $=3.983$ calories.

Le rendement est donc de $\frac{3.9^{83}}{4.448} \times 100=89 \%$.

Aussi l'on peut constater qu'au point de vue de la répartition calorifique, un haut-fourneau marchant à une allure normale, pour la fonte obtenue, ne laisse à peu près rien à désirer.

Mais il n'en est pas moins vrai que son fonctionnement est bien loin d'être facile; il faut une grande expérience et une surveillance de tous les instants pour ne pas avoir de déboires dans la fabrication et l'on ne parvient pas toujours à les éviter! La grande masse de matières portées à une haute température s'oppose à tout arrêt dans la marche du fourneau; de plus elle cause une aggravation dans tout dérangement de l'allure normale. D'un autre côté, lorsque l'on peut constater l'origine d'une mauvaise allure, par l'examen des gaz ou des laitiers, on a beau agir immédiatement, soit sur les charges du lit de fusion, ou sur celles du combustible, il faut attendre que ces matières soient arrivées vers l'ouvrage pour en perçevoir les effets ; ce temps est généralement long, dans tous les cas, plusieurs heures, de sorte que,pendant cette période, le mal s'augmente, et l'on arrive quelquefois, malgré tout, à une situation irréparable.

Il ne faut pas oublier, d'autre part, que le fourneau a souvent coûté de 5 à 700.000 francs, sans les machines et les appareils à chauffer le vent; enfin il ne peut subir aucun arrêt, même pour faire des réparations urgentes. Autrement dit, l'appareil n'a pas une marche élastique et, vu ses grandes dimensions, la consommation de combustible est énorme; dans le fourneau moren du Luxembourg elle serait, par 24 heures de i 6520 kil.

Cet ensemble est donc un bloc essentiellement immobile ; quels que soient les changements économiques de la fabrication, il restera en place, quitte à ne plus être utilisés si ces chargements sont par trop défavorables.

\section{III}

Les desiderata à résoudre par le haut-fourneau électrique doivent donc être :

Io L'emploi de l'énergie électrique de telle manière qu'elle donne la température nécessaire aux réactions de réduction et qu'en même temps elle économise en grande partie ou même supprime complètement le corps réducteur c'est-à-dire le carbone actuellement employé.

$2^{\circ}$ L'obtention d'un rendement calorifique au moins égal à celui du haut-fourneau.

3o L'augmentation de la rapidité de fusion et partant de la production, dans le but d'arriver économiquement à une marche discontinue qui, de ce fait, deviendrait élastique et éviterait la formation des loups entrainant la perte des appareils actuels; de plus, dans ces conditions, le travail de nuit serait probablement supprimé.

$4^{\circ}$ De diminuer, par tonne de matiere produite, le volume intérieur et par suite le volume total du haut-fourneau, conséquemment abaisser dans de grandes limites ses énormes frais de premier établissement. 
50 L'emploi, pour la construction du fourneau, de matériaux réfractaires, mais neutres, c'est-à-dire pouvant passer de la marche acide à la marche basique, ou inversement, sans avoir à craindre les détériorations dues à la formation de matières fusibles lorsque la composition du lit de fusion change.

Le jour où ces desiderala seraient réalisés, l'appareil réducteur des minerais de fer ne serait plus un haut-fourneau. Celui-ci, avec sa masse monumentale, ses récupérateurs extrêmement encombrants, ses immenses machines soufflantes constitue un groupe dont l'ensemble est colossal et le prix de premier établissement hors de proportion avec sa production journalière.

Le four électrique, aux dimensions considérablement réduites et marchant sans l'aide d'accessoires comparables à ceux du haut-fourneau actuel, nous apparaît, au contraire, comme devant être un appareil d'installation rapide et peu coûteuse, et d'adaptation facile aux diverses exigences économiques.

C. Brouzet,

Métallurgiste

Ingénieur civil (E.C.P.)

\section{DU CHOIX DES GIMENTS}

\section{dans les travaux d'aménagagement dess ehutess d'eaul.}

La plupart des industriels a qui tont bâtir » et même bon nombre d'entrepreneurs n'ont jamais, faute d'études spéciales ou de temps, raisonné la question ciment au point de vue technique. Ainsi, rarement on voit dans les cahiers des charges des entreprises privées, un ingénieur imposer des prescriptions spéciales à l'emploi des ciments; tout au plus $\mathrm{y}$ indique-t-on le dosage des mortiers. Presque toujours, c'est l'entrepreneur qui choisit la marque du produit - sur la réputation acquise - et qui, de ce fait, endosse toute la responsabilité inhérente à son initiative. Et nous sommes des premiers à reconnaître qu'industriels, ingénieurs et entrepreneurs, en agissant ainsi, font bien chacun son métier puisque les ouvrages exécutés répondent à leurs fins.

Mais les uns et les autres doivent-ils considérer comme superflue la connaissance des élémentaires notions scientifiques qui, tout en dévoilant le secret de la fabrication des ciments - que d'aucuns s'imaginent perdu depuis les Romains - permettent de concevoir de plus judicieux emplois de matériaux? Le bon produit fait le bon entrepreneur, nous disait très justement l'un d'eux. Il faut donc que celui qui le fait employer, comme celui qui l'emploie, sache apprécier un bon produit et en tirer le meilleur parti possible.

Regardons ce qui se passe aux Ponts-et-Chaussées. Un cahier des charges en 23 articles impose le contròle, par l'Administration, de la fabrication dans l'usine ; la vérification et l'essai après l'arrivée au magasin, la détermination de la densité, de la finesse de mouture, de la composition chimique; des essais sur lă confection des mortiers, sur la durée de la prise, la déformation à froid et à chaud des mortiers, leur résistance à l'arrachement et à l'écrasement, etc... Chaque fois qu'une fourniture doit être faite pour un ouvrage quelconque, un ponceau ou une jetée, le ciment n'est admis sur le chantier que s'il a satisfait aux prescriptions du cahier des charges. Il est certain que le Génie civil, pour ce qui est $d u$ bâtiment, n'a nul besoin de toutes ces précautions et que l'emploi d'une bonne marque est une condition de sécurité suffisante; mais lorsqu'il s'agit, pour dériver un torrent, d'établir des barrages, des canalisations en ciment, c'est à-dire des ouvrages tenant suspendues au flanc des vallées des masses d'eau énormes qu'un défaut de construction peut précipiter sur des villages et y amener la ruine, les conditions de sécurité à remplir sont tout autres, et les auteurs de ces travaux ne sauraient s'entourer de trop de garanties.

Nous nous proposons en cet article, d'expliquer sommairement ce qu'est un ciment, de décrire les particularités distinctives des différentes sortes de ce produit et ainsi permettre aux constructeurs de se rendre compte des qualités ou des défauts du produit qu'ils emploient.

Lorsqu'on analyse un échantillon de ciment en poudre, on trouve qu'il se compose de silice, d'alumine et d'oxyde de fer, d'une part, de chaux et de magnésie d'autre part, et qu'il s'y trouve de l'acide sulfurique. Suivant les proportions relatives de ces éléments combinés entre eux, on a des ciments, soit à prise lente (Portlands), soit à prise prompte (ciments prompts). Ces derniers renferment plus d'alumine que les Portlands.

La théorie de M.LeChâtelier, actuellement admise, montre que les ciments sont essentiellement constitués par des silicates de chaux et des aluminates de chaux; les autres composants : oxyde de fer, sulfate de chaux et magnésie, ne jouent qu'un rôle secondaire. Au contact de l'eau de gâchage, il se produit deux réactions fondamentales : l'une déterminant la rapidité de la prise, l'autre, le durcissenent. La première est l'hydratation plus ou moins rapide de l'aluminate de chaux, suivant les proportions de cet élément; la seconde est le dédoublement du silicate basique de chaux en silicate monocalcique et hydrate de chaux.

Un ciment est d'autant meilleur que toute la chaux qu'il contient peut, pendant la cuisson, se combiner à la silice et à l'alumine, en sorte qu'après la fabrication il ne reste pas de chaux libre dans le produit. Nous dirons plus loin quels sont les graves inconvénients dûs à cette chaux libre et les moyens employés pour atténuer ses effets.

Voici un tableau indiquant entre quelles limites varient les éléments constitutifs des principaux ciments :

\begin{tabular}{|c|c|c|c|}
\hline $\begin{array}{c}\text { Corps } \\
\text { composants. }\end{array}$ & Portlands artificiels. & $\begin{array}{l}\text { Portlands naturels } \\
\text { et ciments } \\
\text { de grappiers. }\end{array}$ & Ciments prompls. \\
\hline Silice............. & 20.30 à 26.10 & 21.30 a 27.50 & $21.70 \div 23.60$ \\
\hline Alumine...$\ldots \ldots$ & $5.20 \times 10.60$ & $1.80 \div 10.02$ & 7.99 a 10.03 \\
\hline Oxyde de fer..... & $2.10 \div 5.30$ & $1.01 \mathrm{a} \quad 4.08$ & 3.71 à 4.31 \\
\hline Chaux ........... & 5812 a 67.31 & 52.50 a 62.70 & 52.68 à 57.40 \\
\hline Magnésie.......... & 0.33 a 2.30 & 0.40 à 3.06 & 1.50 à 3.62 \\
\hline Acide sulfurique ... & 0.26 à 1.78 & 0.55 à 3.70 & 2.10 a 3.56 \\
\hline Perte au fet...... & $\eta \quad D$ & 3.00 à 14.20 & $2.75 a 6.20$ \\
\hline $\begin{array}{r}\text { Eléments non doses } \\
\text { et pertes........ }\end{array}$ & $y$ & ) & 0.11 à 0.35 \\
\hline
\end{tabular}

